Published in final edited form as:

J Proteome Res. 2018 September 07; 17(9): 2978-2986. doi:10.1021/acs.jproteome.8b00166.

\title{
Triflic Acid Treatment Enables LC-MS/MS Analysis of Insoluble Bacterial Biomass
}

\author{
Ana Y. Wang ${ }^{\dagger, \#, ~ P e t e r ~ S . ~ T h u y-B o u n ~}{ }^{\dagger, \#}$, Gregory S. Stupp ${ }^{\dagger}$, Andrew I. Su${ }^{\dagger}$, Dennis W. \\ Wolan ${ }^{*} \dagger$ \\ tDepartment of Molecular Medicine and Department of Integrative Structural and Computational \\ Biology, The Scripps Research Institute, 10550 North Torrey Pines Road, La Jolla, California \\ 92037, United States
}

\begin{abstract}
The lysis and extraction of soluble bacterial proteins from cells is a common practice for proteomics analyses, but insoluble bacterial biomasses are often left behind. Here, we show that with triflic acid treatment, the insoluble bacterial biomass of $\mathrm{Gram}^{-}$and $\mathrm{Gram}^{+}$bacteria can be rendered soluble. We use LC-MS/MS shotgun proteomics to show that bacterial proteins in the soluble and insoluble postlysis fractions differ significantly. Additionally, in the case of $\mathrm{Gram}^{-}$ Pseudomonas aeruginosa, triflic acid treatment enables the enrichment of cell-envelope-associated proteins. Finally, we apply triflic acid to a human microbiome sample to show that this treatment is robust and enables the identification of a new, complementary subset of proteins from a complex microbial mixture.
\end{abstract}

\section{Graphical Abstract}

\footnotetext{
"Corresponding Author: D.W.W.: wolan@ @scripps.edu. Tel.: (858) 784-7936.

\#A.Y.W. and P.S.T.-B. contributed equally.

Author Contributions

A.Y.W., P.S.T.-B., and D.W.W. conceived of the project. P.S.T.-B. and A.Y.W. performed wet-lab experiments and collected MudPIT LC-MS/MS data. A.Y.W., P.S.T.-B., and G.S.S. analyzed tandem LC-MS/MS data. A.Y.W., P.S.T.-B., and G.S.S. contributed to peptide mapping and functional analysis. The manuscript was written through contributions of all authors. All authors have given approval to the final version of the manuscript.

Supporting Information

The Supporting Information is available free of charge on the ACS Publications website at DOI: 10.1021/acs.jproteome.8b00166. Detailed schematic overview of two proteome preparation methods; base-peak chromatograms for microbiome A-supernatant samples; and multiple tables showing the 10 most positively or negatively enriched proteins among sample preparation methods in $P$. aeruginosa, B. subtilis, Jurkat cells, and human microbiome (PDF)

Full list of detected proteins from each LC-MS/MS data set (XLSX)

The authors declare no competing financial interest.
} 


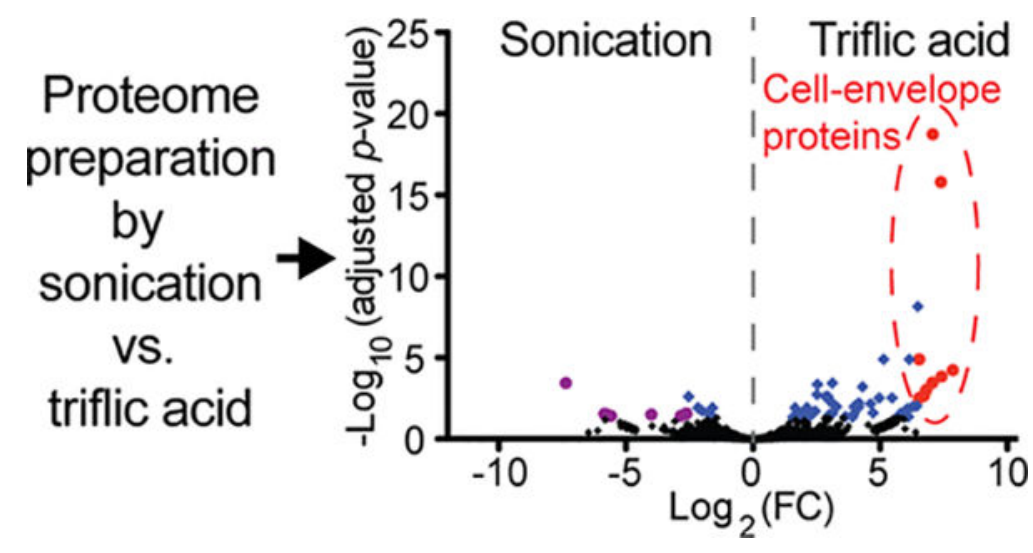

\section{Keywords}

triflic acid; membrane proteins; microbiome; metaproteomics; MudPIT; ComPIL

\section{INTRODUCTION}

Analysis of intracellular proteins by shotgun proteomics requires cells to be lysed and the contents proteolyzed for LC-MS/MS. ${ }^{1-4}$ Simple cell-disruption methods (e.g., detergents, sonication) are sufficient for the full lysis and solubilization of human cells. However, for single bacterial species and complex microbial mixtures (e.g., microbiomes), simple methods of cell disruption yield soluble protein and leave behind large, insoluble, proteolytically resistant pellets. More effective methods of microbial cell disruption ${ }^{5-7}$ utilizing chaotropes, ${ }^{8,9}$ detergents, ${ }^{10}$ bead beating, ${ }^{11}$ sonication, ${ }^{12-15}$ freeze-thawing, ${ }^{16,17}$ aqueous acid/base treatments, ${ }^{18,19}$ organic extraction, ${ }^{20}$ or a combination thereof ${ }^{3,4,21-38}$ have been applied to recalcitrant microbiota isolated from terrestrial, aquatic, and gastrointestinal systems in the past. Our attempts to adapt many of these methods to microbiome samples yielded familiar results, including soluble protein solutions and large insoluble, proteolytically resistant pellets. The size of these remaining pellets lead us to question whether residual protein is trapped in this insoluble matrix and overlooked by downstream analyses. We reasoned that the pellets' resistance to solubilization and proteolysis could partly be due to carbohydrate-rich bacterial macromolecules that include peptidoglycans, lipopolysaccharides, lipotechoic acids, and other cell-envelope constituents that are absent in human cells. ${ }^{39} \mathrm{We}$ therefore sought a method to fragment and separate carbohydrate macromolecules from insoluble bacterial pellets without destroying any putatively associated pellet proteins.

Trifluoromethanesulfonic acid (triflic acid, TA) is used to fully remove glycans from $\mathrm{N}$ linked, O-linked, and glycosaminoglycan-containing glycoproteins while preserving peptide integrity. TA can also deglycosylate $N$-linked glycoproteins, leaving behind a single $N$ linked monosaccharide under highly controlled conditions. ${ }^{40,41}$ This reactivity was recently utilized by the Wu group to map protein $\mathrm{N}$-glycosylation sites of the soluble yeast proteome by LC-MS/MS. ${ }^{42}$ We hypothesized that if insoluble microbial pellets are composed largely 
of carbohydrates, TA could remove these molecules, enabling proteomic analysis of any putatively associated proteins.

Three species with distinct cell envelope morphologies were selected for TA treatment or sonication and downstream LC-MS/MS analysis: (1) Gram ${ }^{-}$Pseudomonas aeruginosa, with a peptidoglycan layer between two membranes; (2) $\mathrm{Gram}^{+}$Bacillus subtilis, with an exposed peptidoglycan layer; and (3) human Jurkat cells - a peptidoglycan-less control. We then TAtreated a human distal gut microbiome sample for LC-MS/MS analysis to evaluate the robustness of this treatment for complex microbial mixtures.

\section{MATERIALS AND METHODS}

\section{Sonication-Based Proteome Preparation of Single Species}

Overnight cultures of $P$. aeruginosa or $B$. subtilis (grown in $5 \mathrm{~mL} 2 \mathrm{XYT}$ media at $37^{\circ} \mathrm{C}$ ) and human Jurkat cells (grown in RPMI-1640 Medium with $10 \%$ heat-inactivated fetal bovine serum) were divided into 100-mg wet pellets. The cells were suspended in $500 \mu \mathrm{L} \mathrm{PBS,} \mathrm{pH}$ 7.4 and sonicated in a Qsonica Q700 sonicator with cup horn attachment at 25\% amplitude for $10 \mathrm{~min}$ at $4{ }^{\circ} \mathrm{C}$. Insoluble cellular debris was removed by centrifugation at $10000 \mathrm{~g}$ for 5 min at room temperature, and the supernatant was carefully removed and filtered through a PD MiniTrap G-25 size exclusion column (GE Life Sciences, No. 28918007) to remove residual, low molecular-weight contaminants. The soluble protein content in the flowthrough (samples designated as A-supernatant, see Supporting Information, Figure 1 for details) was then quantified using the Pierce BCA Protein Assay Kit (Thermo Fisher, No. 23225).

\section{Triflic Acid Proteome Preparation of Single Species}

To prepare the TA-treated samples (samples designated as C-cell), 100-mg (wet pellet) aliquots of $P$. aeruginosa, B. subtilis, and Jurkat cells were suspended in $500 \mu \mathrm{L}$ of PBS, pH 7.4, frozen, and lyophilized overnight. Next, $200 \mu \mathrm{L}$ of 1:9 toluene:TA (TCI, no. T0751, CAS[1493-13-6]) was added to each lyophilized whole-cell sample at $-78^{\circ} \mathrm{C}$ (dry ice/ acetone bath). Tubes containing the solutions were brought to $4{ }^{\circ} \mathrm{C}$ and gently agitated for 30-60 min with intermittent venting until the pellet was fully dissolved. Upon complete solubilization, the solution was refrozen at $-78{ }^{\circ} \mathrm{C}$ (dry ice/acetone bath). The acid was quenched with $600 \mu \mathrm{L}$ of a cold solution of 1:1:3 water:methanol:pyridine (neutralizing solution), and the tubes were inverted several times with frequent venting. The neutralized solution was diluted to $10 \mathrm{~mL}$ with $50 \%$ methanol/water and concentrated to $1 \mathrm{~mL}$ with an Amicon Ultra-15 3K MWCO centrifugal concentrator (Fisher Scientific, No. UFC900324). The sample was diluted to $10 \mathrm{~mL}$ with PBS, $\mathrm{pH} 7.4$, and reconcentrated with a $3 \mathrm{~K}$ MWCO concentrator. The remaining solution and protein were transferred to a microcentrifuge tube and lyophilized. The protein solid was resuspended in $200 \mu \mathrm{L}$ of $5 \mathrm{M}$ urea in $100 \mathrm{mM}$ Tris, pH 8.0 and sonicated until the protein dissolved (see Figure S1 for details). The protein concentration was quantified using the BCA assay.

The remaining insoluble, postsonication pellets from the $B$. subtilis and $P$. aeruginosa proteome preparations were resuspended in PBS pH 7.4, frozen, lyophilized, and TA-treated 
with the same methods as described for the C-cell preparations (samples are designated as B-pellet, Figure S1).

\section{Sonication and Triflic Acid Preparations of Microbiome Samples}

Fecal samples were suspended in PBS and mixed by vortexing until full suspension and uniformity. Samples were centrifuged at $100 \mathrm{~g}$ for $1 \mathrm{~min}$, and the cloudy supernatant was filtered through a 70- $\mu \mathrm{m}$ nylon cell strainer (Fisher Scientific, no. 08-771-2) to remove large particles. The microbial cells were pelleted by centrifugation at $8000 \mathrm{~g}$ for $5 \mathrm{~min}$, rinsed twice with PBS, pH 7.4, and divided into 100-mg cell pellets (wet weight) for each sample. For C-cell preparation, microbial cell pellets were suspended in PBS, $\mathrm{pH} 7.4$, frozen, and lyophilized. First, $300 \mu \mathrm{L}$ of $1: 9$ toluene:TA was added to each sample at $-78^{\circ} \mathrm{C}$ (dry ice/ acetone bath). The sample temperature was increased to $4{ }^{\circ} \mathrm{C}$ and gently agitated for $30-60$ min at $4{ }^{\circ} \mathrm{C}$ with intermittent venting. After the cell pellet was completely liquefied, the solution was frozen at $-78{ }^{\circ} \mathrm{C}$ (dry ice/acetone bath) and quenched with $900 \mu \mathrm{L}$ of a cold solution of 1:1:3 water:-methanol:pyridine. The tubes were inverted with frequent venting until the frozen pellet was dissolved. This solution was diluted to $10 \mathrm{~mL}$ with $50 \%$ methanol/water, concentrated to $1 \mathrm{~mL}$ with a $3 \mathrm{~K}$ MWCO centrifugal concentrator, and then diluted and reconcentrated twice more with $100 \mathrm{mM}$ Tris $\mathrm{HCl}, \mathrm{pH}$ 8.0. The remaining protein suspension was transferred to a $1.5 \mathrm{~mL}$ microcentrifuge tube, frozen, and lyophilized. The protein was resuspended in $5 \mathrm{M}$ urea in $100 \mathrm{mM}$ Tris $\mathrm{HCl}, \mathrm{pH} 8.0$ and sonicated until completely dissolved, and the protein concentration was quantified using the BCA assay (Figure S1). For B-pellet preparation, the pellet remaining after sonication was TA-treated, and protein was extracted similarly to single-species B-pellet samples (Figure S1). For the microbiome A-supernatant sample preparation, soluble proteins were collected after microbiome sonication, centrifugation, and size exclusion purification (PD MiniTrap G-25) similarly to single-species A-supernatant samples (Figure S1). However, an additional step was required that included the collected supernatant to be frozen, lyophilized overnight, and then TA-treated (as for B-pellet and C-cell microbiome fractions).

\section{Preparation of Samples for LC-MS/MS Analysis}

Initially, $100 \mu \mathrm{g}$ of protein per sample suspended in $120 \mu \mathrm{L}$ of $100 \mathrm{mM}$ Bicine, $5 \mathrm{M}$ urea, $\mathrm{pH}$ 8.0 were treated with tris(2-carboxyethyl)phosphine (TCEP) (Thermo Fisher, No. 20490) to a final concentration of $5 \mathrm{mM}$ and incubated for $15 \mathrm{~min}$, followed by chloroacetamide (Sigma-Aldrich, No. 201-174-2, CAS[79-07-2]) treatment at a final concentration of $25 \mathrm{mM}$ for $15 \mathrm{~min}$ in the dark. The mixture was then diluted to a final volume of $500 \mu \mathrm{L}$ with trypsin buffer (100 mM Tris HCl, pH 8.0 with $1 \mathrm{mM}$ calcium chloride), and $2 \mu \mathrm{g}$ of trypsin (Promega, No. V5111) was incubated with each sample overnight at $37{ }^{\circ} \mathrm{C}$ under agitation. After incubation, samples were treated with $25 \mu \mathrm{L}$ of formic acid, and the samples were centrifuged at $12000 \mathrm{~g}$ for $5 \mathrm{~min}$. Lastly, $475 \mu \mathrm{L}$ of the topmost liquid layer was separated and stored at $-20^{\circ} \mathrm{C}$.

\section{LC-MS/MS MudPIT Data Collection}

The trypsin-digested peptides of single-species samples were loaded onto a biphasic column (250 $\mu \mathrm{m}$ fused silica, Thomas Scientific, No. 2713X55), with $3 \mathrm{~cm}$ of $5 \mu \mathrm{m}$ Aqua C18 resin (Phenomenex, 00G-4299-E0) followed by $3 \mathrm{~cm}$ of Partisphere strong cation exchange 
(SCX) resin. The analytical column was made by pulling $100 \mu \mathrm{m}$ fused silica (Thomas Scientific, No. 2713X83) to a $5 \mu \mathrm{m}$ tip with a micropipette puller (Sutter Instrument Company, Model P-2000) and packed with $10 \mathrm{~cm}$ of $5 \mu \mathrm{m}$ Aqua C18 resin. The sample and analytical columns were joined using a zero-dead volume union (Waters).

Multidimensional protein identification technology (Mud-PIT) ${ }^{43,44}$ tandem mass spectrometry (LC-MS/MS) was performed using a Thermo Finnigan LTQ attached to an Agilent 1200 series quaternary pump. The pump was set at $200 \mu \mathrm{L} / \mathrm{min}$, and peptides were eluted at a rate of $250 \mathrm{~nL} / \mathrm{min}$, using a 6-step MudPIT program. The first step began with 2.5 min of $100 \%$ buffer $\mathrm{A}\left(95 \% \mathrm{H}_{2} \mathrm{O}, 5 \%\right.$ acetonitrile, $0.1 \%$ formic acid), followed by $30 \mathrm{~min}$ of $15 \%$ buffer $\mathrm{B}\left(20 \% \mathrm{H}_{2} \mathrm{O}, 80 \%\right.$ acetonitrile, $0.1 \%$ formic acid) and $85 \%$ buffer A. Buffer B was then increased over 20 min to $100 \%$ before ending with 20 min of $100 \%$ buffer A. Each salt step began with 1 min of $100 \%$ buffer A, followed by a 4-min salt pulse with various concentrations of buffer $\mathrm{C}\left(500 \mathrm{mM}\right.$ ammonium acetate, $95 \% \mathrm{H}_{2} \mathrm{O}, 5 \%$ acetonitrile, $0.1 \%$ formic acid), then $5 \mathrm{~min}$ of $100 \%$ buffer $\mathrm{A}$, followed by a $105 \mathrm{~min}$ gradient from 5\%-65\% buffer $\mathrm{B}$, and ending with $5 \mathrm{~min}$ of $100 \%$ buffer $\mathrm{A}$. The salt pulses of buffer $\mathrm{C}$ were as follows: $10 \%, 25 \%, 50 \%, 80 \%, 100 \%$, with buffer A as the diluent. The MS was operated with the following settings: MS1 mass range of 300-2000, 5 precursor ions selected for tandem MS per scan cycle, intensity threshold of 500 for triggering MS2, relative collision energy $35 \%$, and dynamic exclusion set to exclude after two times if occurs within $30 \mathrm{~s}$ with an exclusion duration of $20 \mathrm{~s}$.

For microbiome samples, $40 \mu \mathrm{L}$ of the peptide solution from "Proteome sample preparation for LC-MS/MS" was dried using a SpeedVac concentrator and desalted using $10 \mu \mathrm{L}$ ZipTips $\mathrm{C}_{18}$ (Millipore). The resulting peptide solution dried using the SpeedVac concentrator and was analyzed with a Thermo Fisher Orbitrap Fusion Tribrid mass spectrometer coupled to a nLC 1000 system in the Proteomics Core at TSRI Florida.

The dry peptides were reconstituted in $40 \mu \mathrm{L}$ of $0.1 \%$ formic acid in water, and $10 \mu \mathrm{L}$ of the peptide solution were used for LC-MS/MS analysis. Peptides were online eluted onto an analytical reversed-phase column $(0.075 \times 150 \mathrm{~mm}$ Acclaim PepMap RLSC nano Viper, Thermo Fisher) at $300 \mathrm{~nL} / \mathrm{min}$ with the following gradients, using buffer $\mathrm{A}(0.1 \%$ formic acid in $\mathrm{H}_{2} \mathrm{O}$ ) as the diluent: 5-25\% buffer $\mathrm{B}\left(80 \%\right.$ acetonitrile, $0.1 \%$ formic acid, $20 \% \mathrm{H}_{2} \mathrm{O}$ ) in $160 \mathrm{~min}, 25-44 \%$ buffer B in $80 \mathrm{~min}, 44-80 \%$ buffer B in $10 \mathrm{~s}, 80 \%$ buffer B for $5 \mathrm{~min}$, $80-5 \%$ buffer B in $10 \mathrm{~s}$, and 5\% B for $20 \mathrm{~min}$. The MS was operated with the following settings: MS1 scan range of 380-1400 m/z with a mass tolerance of $10 \mathrm{ppm}$ and $120 \mathrm{~K}$ resolution using Orbitrap detection, data-dependent MS/MS mode at the maximum speed with precursor priority of the most intense ions, HCD fragmentation with normalized collision energy of 30\%, 1.0E4 AGC Target intensity threshold for MS2, 2-8 charge states included in screening parameters, a mass resolution of $30 \mathrm{~K}$ for MS2, and dynamic exclusion set to exclude after two times if occurs within $30 \mathrm{~s}$ with an exclusion duration of $20 \mathrm{~s}$. All LC-MS/MS data have been deposited to the ProteomeXchange Consortium (http:// proteomecentral.proteomexchange.org) via the PRIDE partner repository with the project accession identifier PXD009004. ${ }^{45}$ 


\section{Peptide and Protein Identification}

For the MS data of single-species samples, rawXtract 1.9.9.2 was used to extract precursor and fragmentation ion data in MS2 format from Xcalibur RAW files. Integrated Proteomics Pipeline (IP2, Integrated Proteomics Applications, Inc. San Diego, CA) was utilized for protein identification and quantification analyses, using PROLUCID/Sequest ${ }^{46,47}$ and DTASelect. ${ }^{47,48}$ Tandem mass spectra were extracted into the MS2 file format from the Xcalibur RAW files via RawXtract 1.9.9.2 (http://fields.scripps.edu/yates/wp/?page_id=17). The MS2 spectra were scored against the protein library of each respective species from Uniprot proteome IDs, UP000001570 (Bacillus subtilis 168), UP000002438 (Pseudomonas aeruginosa PA01), and UP000005640 (Homo sapiens), which include the reversed sequence for each entry in the original database, ${ }^{49}$ using PROLUCID/Sequest. The settings for peptide scoring include: (1) differential modifications of oxidized methionine (+15.9949 Da) and GlcNAc-tagged asparagine (+203.0794 Da), (2) a static modification for alkylated cysteine residues (+57.02146 Da), (3) precursor/peptide mass tolerance of $3 \mathrm{Da}$, and (4) a fragment mass tolerance of $600 \mathrm{ppm}$. The search space included half- and fully tryptic peptide candidates with two missed cleavage events and required a minimum of two peptides per protein. The ProLUCID search results were assembled and filtered using the DTASelect program. Spectral counts (SC) determined from this pipeline were used as the basis of our quantitative analyses. Venn diagrams were generated on the basis of the presence or absence of one or more SC in each protein.

The human microbiome samples' MS2 spectra were scored using Blazmass 0.9993 against the peptides of the Comprehensive Protein Identification Library (ComPIL) database. ${ }^{50}$ Blazmass and ComPIL source code are open source (https://github.com/sandipchatterjee/ blazmass_compil). Settings for peptide scoring include a variable modification of oxidized methionine $(+15.9949 \mathrm{Da})$, a static modification for alkylated cysteine residues $(+57.02146$ $\mathrm{Da})$, a precursor mass tolerance of $10 \mathrm{ppm}$, and a fragmentation ion tolerance of $50 \mathrm{ppm}$. DTASelect 2.1.3 (http://fields.scripps.edu/yates/wp/?page_id=17) was used for filtering with the requirements of two peptides per protein and a protein FDR of $1 \%$.

The source code for this analysis is available online (https://github.com/stuppie/triflic/tree/ master/H1). Protein clustering, cluster taxonomy, and gene ontology (GO) term annotations were performed as previously described. ${ }^{50-52}$ Protein loci were mapped to protein clusters with ComPIL using a sequence identity threshold of $70 \%$. A protein cluster was annotated with all GO terms associated with any domain for all possible proteins in that cluster. Any GO terms that were parents ("is a" or "part of" relationships) of other GO terms in that protein cluster were removed. Annotations were generated from InterProScan v5 (version 5.8-49.0).

\section{Differential Analysis of Detected Proteins}

Protein differential analysis was performed using DESeq2 1.16.1, which allows for testing for differential expression using SC data and provides methods designed for taking into account overdispersion, features with low counts, and experiments with low numbers of replicates. ${ }^{53}$ This program has previously been applied to proteomic studies. ${ }^{51,52,54}$ Briefly, count data is modeled using the negative binomial distribution, and the mean-variance 
relationship is estimated. Variance is estimated using an information sharing approach, whereby a single feature's (or protein cluster's) variance is estimated by taking into account variances of other protein clusters measured in the same experiment. Feature significance calling and ranking is performed using estimated effect sizes, which takes into account the logarithmic fold change (LFC) of a protein locus between enriched and unenriched samples and the noisiness of the LFC estimate. Replicates were not merged, but were treated as replicates for the differential analysis. The PCA plot was generated using DESeq2 on the variance-stabilized (i.e., transformed) SC for each protein or protein locus. All volcano plots were created with Prism v5 (GraphPad, Inc.). SC calculated for the clusters are the sum of the SC of all proteins within the cluster identified by LC-MS/MS. Tables S1-S16 highlight the top 10 most positively or negatively differentially detected proteins among sample preparation methods. A full list of all detected proteins is provided in the Supporting Information data set.

\section{GO Slim Terms for Microbiome Sample Analysis}

Each protein locus was mapped to $\mathrm{GO}^{55,56}$ terms associated with any domain of all possible proteins within each locus identified by LC-MS/MS. A GO Slim mapping file was generated by downloading GO mapping files from Uniprot-GOA (released June 6, 2017; ftp:// ftp.ebi.ac.uk/pub/databases/GO/goa/UNIPROT/goa_uniprot_gcrp.gaf.gz) and the Gene Ontology (release June 19, 2017, http://purl.obolibrary.org/obo/go.obo). A simplified version of the $\mathrm{GO}$ (GO slim) was created using Owltools (https://github.com/owlcollab/ owltools), and it consisted of the terms within the Cellular Component (CC) ontology, including membrane (GO:0016020), cell (GO:0005623), cytoplasm (GO:0005737), organelle (GO:0043226), cell periphery (GO:0071944), and the ontology CC (GO:0005575) itself. The resulting dictionary generated contains the Uniprot ID of each protein as the key, and the value is the list of GO CC terms annotated to that protein cluster.

\section{Assignment and Quantification of Cellular Component GO Annotations}

$\mathrm{CC}$ annotations associated with each protein cluster were retrieved from the generated dictionary, and the total SC of peptides within each cluster were summed. The count contributed by each protein cluster to a $\mathrm{CC}$ annotation was calculated by dividing the total sum of the SC of each cluster by the number of $\mathrm{CC}$ annotations associated with that cluster, which controls for inflation of values due to annotation biases (i.e., more- versus lessstudied/annotated proteins).

The cellular localizations in Figure 2C were derived from the $\mathrm{CC}$ annotations we retrieved for the protein clusters identified by LC-MS/MS. Four categories were created by binning together the CC terms as follows: (1) Membrane \& Cell Envelope: membrane (GO:0016020), cell periphery (GO:0071944); (2) Cytosol: cytoplasm (GO:0005737); (3) Cell: cell (GO:0005623), organelle (GO:0043226), cellular component (GO:0005575); and (4) No CC Annotation: no CC annotations listed for the protein. 


\section{RESULTS AND DISCUSSION}

$P$. aeruginosa, $B$. subtilis, Jurkat cells, and a healthy human microbiome sample were partitioned into two sets. Samples in the first set were lysed by sonication ${ }^{52}$ (A-supernatant), clarified by centrifugation, and prepared for LC-MS/MS analysis (Figure 1). While Jurkat cells fully dissolved, bacterial samples yielded yellow supernatants and insoluble pellets. The remaining $P$. aeruginosa, $B$. subtilis, and microbiome pellets (B-pellet) were TA-treated and prepared for LC-MS/MS analysis. ${ }^{43,44}$ The second set of samples were TA-treated directly and prepared for LC-MS/MS (C-cell) (Figure 1). TA treatment completely dissolved whole bacterial cells and the previously insoluble bacterial pellets left after sonication. Importantly, TA treatment yielded material that is readily solubilized after trypsin digestion or detergent/chaotrope treatment, postworkup. Substantial differences in the compositions of the detected proteomes of each treatment group were observed by LC-MS/MS (see Supporting Information data set). Here, we highlight the proteins with the greatest differences in abundance due to proteome preparation methods.

\section{TA Treatment of $P$. aeruginosa Markedly Increases Cell-Envelope-Associated Protein Detection}

There were striking differences between A-supernatant, B-pellet, and C-cell data sets for $P$. aeruginosa. 2360 proteins were collectively identified, with 1118 shared proteins and 234242 proteins uniquely detected by specific treatments (Figure 2). Comparison of Asupernatant and $\mathrm{C}$-cell showed that the detected quantities of 78 proteins changed significantly with an absolute $\log _{2}$ fold-change $\left(\left|\log _{2} \mathrm{FC}\right|\right)>1$ (Figure 3A). Overall, 60/78 proteins experienced enrichments of $\log _{2} \mathrm{FC} 1.58$ to 11.8 with TA treatment. Of the 10 mostshifted proteins, 9 are cell envelope-associated (Table S1). Among the most positively enriched proteins were two envelope-associated, uncharacterized proteins (Q9HVI2, Q9HVK6), large-conductance mechanosensitive channel (Q9HVH7), and outer membrane (OM) porin $\mathrm{F}$ (P13794), with a $\log _{2} \mathrm{FC}$ of 11.8 and 7.86, 7.42, and 7.08, respectively. Conversely, 18 proteins were negatively enriched in the C-cell data set, with 5 of the 10 most shifted proteins annotated as cytoplasmic, 2 as periplasmic, and 3 with unknown localizations (Supporting Information, Table 2). These negatively enriched cytoplasmic proteins include formyltetrahydrofolate deformylase (Q9HW87) and pterin-4-alphacarbinolamine dehydratase ( $\mathrm{P} 43335$ ), with $\log _{2} \mathrm{FC}$ values of -5.74 and -4 , respectively.

The detected quantities of 265 P. aeruginosa proteins shifted significantly between Asupernatant and B-pellet (Figure 3A). In total, 209/264 proteins were positively enriched in B-pellet, with $\log _{2} \mathrm{FC}$ from 1.25 to 9.03 with TA treatment (Table S3). All of the 10 mostenriched proteins are annotated as envelope-associated, and 7 of these proteins were not detected in A-supernatant, including PhoP/Q and low $\mathrm{Mg}^{2+}$ inducible OM protein $\mathrm{H} 1$ (G3XD11), two probable OM proteins (Q9I456, Q9HVI2), motility protein FimV (Q9HZA6), lipid A deacylase PagL (Q9HVD1), membrane-bound lysozyme inhibitor (Q9I574), and OM protein assembly factor BamB (Q9HXJ7). 56/265 proteins were negatively enriched in B-pellet, ranging from $\log _{2} \mathrm{FC}-1.09$ to -7.02 (Figure $3 \mathrm{~A}$ ). Of the 10 most shifted proteins, 4 are cytoplasmic, 4 are periplasmic, and 2 are of unknown localization (Table S4). For $\mathrm{Gram}^{+} P$. aeruginosa, TA treatment favors the LC-MS/MS 
detection of envelope-associated proteins and clearly demonstrates that the postsonication insoluble pellet contains important proteomic information missed with sonication-only preparations.

\section{Preparation of B. subtilis by Sonication and TA Treatment Preparations Yield Complementary Proteome Information}

The analysis of $\mathrm{Gram}^{+}$B. subtilis identified a total of 1680 proteins, with 725 detected in all three sample preparations and 211, 165, and 143 unique proteins identified in A-supernatant, B-pellet, and C-cell, respectively (Figure 2). Between A-supernatant and C-cell data sets, 380 proteins were differentially detected, and 206/380 were found in greater abundance due to TA treatment, ranging from $\log _{2} \mathrm{FC}$ enrichments of 1.02 to 10.2 (Figure 3B). Six of the 10 most-enriched proteins are cytoplasmic, including cyclo(L-leucyl-L-leucyl) synthase (O34351) and dimodular nonribosomal peptide synthase (P45745), and four are envelopeassociated, including the pulcherriminic acid synthase (O34926) and tRNA nuclease WapA (Q07833) (Table S5). 174/380 proteins were negatively enriched in C-cell compared to Asupernatant. 3-Ketoacyl-CoA thiolase (O32177) was the most differentially detected protein with a $\log _{2} \mathrm{FC}$ of -8.63 in C-cell (Table S6).

Differences between B. subtilis A-supernatant and B-pellet data sets were pronounced. 482 proteins were differentially detected, and 216/482 were positively enriched in B-pellet, with $\log _{2} \mathrm{FC}$ from 1.05 to 11.75 (Figure 3B). Similar to the comparison between A-supernatant and C-cell, a mixture of predicted cytoplasmic and envelope-associated proteins were differentially detected (Table S7). 266/482 differentially detected proteins were negatively enriched in B-pellet ( $\log _{2} \mathrm{FC}-1.11$ to -9.63$)$, with glucose starvation-inducible protein B (P26907) as the most-changed (Table S8). In contrast to P. aeruginosa, most TA treatmentenriched $B$. subtilis proteins are not annotated as envelope-associated. However, the subcellular locales of $B$. subtilis proteins are not well annotated in general; thus, it is unclear in this case whether TA treatment broadly enables the enrichment of envelope-associated proteins. What is clear, however, is that sonication and TA treatment of B. subtilis resulted in data sets with complementary proteome information.

\section{Limited Differences in Jurkat Cell Data between Sonication and TA Treatment Preparations}

A total of 6659 proteins were detected from Jurkat cells, with 2741 exclusive to Asupernatant, 962 in C-cell only, and 2956 common proteins (Figure 2). Seventeen proteins were differentially detected between the A-supernatant and C-cell preparations (Figure 3C). 5/17 were positively enriched, ranging from $\log _{2} \mathrm{FC} 3.05$ to 6.63 in the C-cell data set, and consisted of nucleic acid-binding proteins (Table S9). Conversely, 12/17 proteins were negatively shifted, ranging from $\log _{2} \mathrm{FC}-2.50$ to -7.67 in C-cell, including cytoskeleton proteins and four isoforms of pyruvate kinase (Table S10). Overall, few Jurkat proteins with significant changes in detection were found; most proteins exclusive to A-supernatant versus C-cell were of low abundance. In addition, we suspect that TA treatment could be dissociating $N$-linked nucleobases from their sugar-phosphate backbones, thus disaggregating protein-nucleic acid complexes that are resistant to proteolytic cleavage under normal conditions. This, in turn, could explain the enrichment in detected nucleic acid binding proteins with TA treatment. 


\section{TA Treatment of Human Gut Microbiome Samples Greatly Improves Protein Detection}

Metaproteomic analysis of a healthy human gut microbiome yielded a total of 8083 unique protein clusters ${ }^{50}$ collectively among the treatment methods, with an average of 22203 matched spectra per run. Principal component analysis (Figure 4A) and protein overlap demonstrate that B-pellet (7021 clusters) and C-cell (6796 clusters) data sets are most similar to each other, and both differ in detectable protein composition from A-supernatant (4569 clusters) (Figures 4B,C) ${ }^{16}$ Of the 8083 protein clusters identified, 5240 were differentially detected between A-supernatant and C-cell, with adjusted $p$-value $<0.05$ and $\mid$ $\log _{2}$ (FC) $\mid>1$ (Figure 5A). 3720/5240 differentially detected protein clusters were positively enriched in C-cell, and several of the most-enriched proteins included elongation factor Tu from a range of bacteria (Table S11). Conversely, 1520/5240 clusters diminished with respect to TA treatment (Table S12). 5180 protein clusters were differentially detected between A-supernatant and B-pellet with adjusted $p$-value $<0.05$ and $\left|\log _{2} \mathrm{FC}\right|>1$ (Figure 5B). 3722/5180 were positively enriched with TA treatment, and 7 of the top 10 protein clusters were the same as those enriched in the C-cell versus A-supernatant (Table S13). $1458 / 5180$ proteins were negatively enriched with TA treatment and vary in composition and parental bacterial species (Table S14). Interestingly, only 786 protein clusters were differentially detected between B-pellet (456/786) and C-cell (330/786) (Figure 5C), and two of the most positively enriched clusters in B-pellet were human (Tables S15 and S16). Surprisingly, additional human proteins were identified in the B-pellet data set despite extensive washing prior to bacterial lysis. Though TA treatment enables the detection of many new microbiome proteins, our subcellular localization analysis is limited by the poorly characterized nature of the gut microbiome's proteome (Figures 5D-G) ${ }^{55,56}$

One of the most remarkable outcomes of our investigation was the impact of TA treatment on the microbiome A-supernatant fraction. LC-MS/MS analysis of the A-supernatant microbiome prepared similarly to the other bacterial and Jurkat cell A-supernatant samples returned 0 protein cluster matches despite RAW files of adequate size and chromatographic quality (Figure S2). This is in sharp contrast to the B-pellet and C-cell microbiome data set analyses that returned thousands of protein cluster matches. We hypothesized that the abundant unmatched features in microbiome A-supernatant could be nonpeptide contaminants that are minimized in B-pellet and C-cell preparations by TA treatment. To test this hypothesis, we TA-treated the same microbiome A-supernatant sample and gratifyingly observed >2000 protein cluster matches after LC-MS/MS analysis, a conspicuous difference compared with the 0 protein cluster matches we observed before TA treatment.

Problems related to ion suppression and signal interference by nonpeptide contaminants in protein preparations have been documented and addressed in previous studies. 17,19-21,23,25,28,32,36,38 For example, with regard to metaproteomic surveys of the soil microbiota, humic acid-type substances have been identified as prominent interfering contaminants. Humic acids have diverse chemical structures and are particularly difficult to completely remove. ${ }^{38}$ For human microbiome samples, it is unclear at this time whether interfering compounds fit a particular chemical profile, but it appears that TA treatment markedly ameliorates their impact on microbiome analysis. 


\section{CONCLUSIONS}

Use of TA in the preparation of bacteria dramatically impacts the detectable proteome. Many proteins were uniquely detected by both sonication and TA treatment, clearly demonstrating the complementarity of TA treatment to established preparation methods. For the $\mathrm{Gram}^{-} P$. aeruginosa, TA treatment enables the enrichment of envelope-associated proteins by LCMS/MS. For the $\mathrm{Gram}^{+}$B. subtilis, a profound enrichment of many proteins is observed, including many envelope-associated proteins; however, there are also many proteins that only appear to be detected with sonication, demonstrating the complementarity of TA treatment to other preparation methods. Finally, TA treatment not only appears robust for a microbiome sample, but it also enabled the analysis of supernatant prepared by sonication that otherwise yielded a null data set. We anticipate that TA treatment will have broad applicability for the analysis of complex microbial systems.

\section{Supplementary Material}

Refer to Web version on PubMed Central for supplementary material.

\section{ACKNOWLEDGMENTS}

We thank Dr. J. Yates, Dr. J. Moresco, and Dr. J. Diedrich for technical assistance with mass spectrometry instrumentation; Dr. M. Perego for providing the $B$. subtilis strain. We also gratefully acknowledge financial support from The Scripps Research Institute, Boehringer Ingelheim (to D.W.W. and A.I.S.), U54GM114833 (to A.I.S.), and the U.S. Environmental Protection Agency STAR Predoctoral Fellowship FP917296-01-0 (to P.S.T.-B.)

\section{REFERENCES}

(1). Kolmeder CA; de Vos WM Metaproteomics of our microbiome - developing insight in function and activity in man and model systems. J. Proteomics 2014, 97, 3-16. [PubMed: 23707234]

(2). Moore SM; Hess SM; Jorgenson JW Extraction, enrichment, solubilization, and digestion techniques for membrane proteomics. J. Proteome Res 2016, 15 (4), 1243-1252. [PubMed: 26979493]

(3). Tanca A; Abbondio M; Palomba A; Fraumene C; Manghina V; Cucca F; Fiorillo E; Uzzau S Potential and active functions in the gut microbiota of a healthy human cohort. Microbiome 2017, 5 (1), 79. [PubMed: 28709472]

(4). Zhang X; Li L; Mayne J; Ning Z; Stintzi A; Figeys D Assessing the impact of protein extraction methods for human gut metaproteomics. J. Proteomics 2017, 180, 120-127. [PubMed: 28705725]

(5). Keller M; Hettich R Environmental proteomics: a paradigm shift in characterizing microbial activities at the molecular level. Microbiol. Mol. Biol. Rev 2009, 73, 62-70. [PubMed: 19258533]

(6). Bastida F; Moreno JL; Nicolas C; Hernandez T; Garcia C Soil metaproteomics: a review of an emerging environmental science. Significance, methodology and perspectives. Eur. J. Soil. Sci 2009, 60, 845-859.

(7). Wang D-Z; Kong L-F; Li Y-Y; Xie Z-X Environmental microbial community proteomics: status, challenges and perspectives. Int. J. Mol. Sci 2016, 17, 1275.

(8). Verberkmoes NC; Russell AL; Shah M; Godzik A; Rosenquist M; Halfvarson J; Lefsrud MG; Apajalahti J; Tysk C; Hettich RL; Jansson JK Shotgun metaproteomics of the human distal gut microbiota. ISME J. 2009, 3, 179-189. [PubMed: 18971961]

(9). Mahowald MA; Rey FE; Seedorf H; Turnbaugh PJ; Fulton RS; Wollam A; Shah N; Wang C; Magrini V; Wilson RK; Cantarel BL; Coutinho PM; Henrissat B; Crock LW; Russell A; Verberkmoes NC; Hettich RL; Gordon JI Characterizing a model human gut microbiota 
composed of members of its two dominant bacterial phyla. Proc. Natl. Acad. Sci. U. S. A 2009, 106, 5859-5864. [PubMed: 19321416]

(10). Sharma R; Dill BD; Chourey K; Shah M; VerBerkmoes NC; Hettich RL Coupling a detergent lysis/cleanup methodology with intact protein fractionation for enhanced proteome characterization. J. Proteome Res 2012, 11, 6008-6018. [PubMed: 23126408]

(11). Kolmeder CA; de Been M; Nikkila J; Ritamo I; Matto J; Valmu L; Salojarvi J; Palva A; Salonen A; de Vos WM Comparative metaproteomics and diversity analysis of human intestinal microbiota testifies for its temporal stability and expression of core functions. PLoS One 2012, 7 , e29913. [PubMed: 22279554]

(12). Ogunseitan OA Direct extraction of catalytic proteins from natural microbial communities. J. Microbiol. Methods 1997, 28, 55-63.

(13). Abram F; Su W-L; Wiedmann M; Boor KJ; Coote P; Botting C; Karatzas KAG; O’Byrne CPO Proteomic analyses of a Listeria monocytogenes mutant lacking $\sigma^{\mathrm{B}}$ identify new components of the $\sigma^{\mathrm{B}}$ regulon and highlight a role for $\sigma^{\mathrm{B}}$ in the utilization of glycerol. Appl. Environ. Microbiol 2008, 74, 594-604. [PubMed: 18065622]

(14). Benndorf D; Vogt C; Jehmlich N; Schmidt Y; Thomas H; Woffendin G; Shevchenko A; Richnow $\mathrm{H}-\mathrm{H}$; von Bergen M Improving protein extraction and separation methods for investigating the metaproteome of anaerobic benzene communities within sediments. Biodegradation 2009, 20, 737-750. [PubMed: 19381451]

(15). Tang Y; Underwood A; Gielbert A; Woodward MJ; Petrovska L Metaproteomics analysis reveals the adaptation process for the chicken gut microbiota. Appl. Environ. Microbiol 2014, 80, 478 485. [PubMed: 24212578]

(16). Ogunseitan OA Direct extraction of proteins from environmental samples. J. Microbiol. Methods 1993, 17, 273.

(17). Singleton I; Merrington G; Colvan S; Delahunty JS The potential of soil protein-based methods to indicate metal contamination. Appl. Soil. Ecol 2003, 23, 25-32.

(18). Schulze WX; Gleixner G; Kaiser K; Guggenberger G; Mann M; Schulze E-D A proteomic fingerprint of dissolved organic carbon and of soil particles. Oecologia 2005, 142, 335-343. [PubMed: 15449171]

(19). Benndorf D; Balcke GU; Harms H; von Bergen M Functional metaproteome analysis of protein extracts from contaminated soil and groundwater. ISME J. 2007, 1, 224-234. [PubMed: 18043633]

(20). Carpentier SC; Witters E; Laukens K; Deckers P; Swennen R; Panis B Preparation of protein extracts from recalcitrant plant tissues: an evaluation of different methods for two-dimensional gel electrophoresis analysis. Proteomics 2005, 5, 2497-2507. [PubMed: 15912556]

(21). Wilmes P; Bond PL The application of two-dimensional polyacrylamide gel electrophoresis and downstream analyses to a mixed community of prokaryotic microorganisms. Environ. Microbiol 2004, 6, 911-920. [PubMed: 15305916]

(22). Ram RJ; VerBerkmoes NC; Thelen MP; Tyson GW; Baker BJ; Blake RC; Shah M; Hettich RL; Banfield JF Community proteomics of a natural microbial biofilm. Science 2005, 308, 19151920. [PubMed: 15879173]

(23). Pierre-Alain M; Christophe M; Severine S; Houria A; Philippe L; Lionel R Protein extraction and fingerprinting optimization of bacterial communities in natural environment. Microb. Ecol 2007, 53, 426-434. [PubMed: 16944344]

(24). Klaassens ES; de Vos WM; Vaughan EE Metaproteomics approach to study the functionality of the microbiota in the human infant gastrointestinal tract. Appl. Environ. Microbiol 2007, 73, 1388-1392. [PubMed: 17158612]

(25). Chen S; Rillig MC; Wang W Improving soil protein extraction for metaproteome analysis and glomalin-related soil protein detection. Proteomics 2009, 9, 4970-4973. [PubMed: 19743425]

(26). Wisniewski JR; Zougman A; Nagaraj N; Mann M Universal sample preparation method for proteome analysis. Nat. Methods 2009, 6, 359-362. [PubMed: 19377485]

(27). Abram F; Gunnigle E; O'Flaherty V Optimisation of protein extraction and 2-DE for metaproteomics of microbial communities from anaerobic wastewater treatment biofilms. Electrophoresis 2009, 30, 4149-4151. [PubMed: 19960479] 
(28). Chourey K; Jansson J; VerBerkmoes N; Shah M; Chavarria KL; Tom LM; Brodie EL; Hettich RL Direct cellular lysis/protein extraction protocol for soil metaproteomics. J. Proteome. Res 2010, 9, 6615-6622. [PubMed: 20954746]

(29). Bastida F; Algora C; Hernandez T; Garcia C Feasibility of a cell separation-proteomic based method for soils with different edaphic properties and microbial biomass. Soil Biol. Biochem 2012, 45, 136-138.

(30). Schneider T; Keiblinger KM; Schmid E; Sterflinger-Gleixner K; Ellersdorfer G; Roschitzki B; Richter A; Eberl L; Zechmeister-Boltenstern S; Riedel K Who is who in litter decomposition? Metaproteomics reveals major microbial players and their biogeochemical functions. ISME J. 2012, 6, 1749-1762. [PubMed: 22402400]

(31). Fouts DE; Pieper R; Szpakowski S; Pohl H; Knoblach S; Suh M-J; Huang S-T; Ljungberg I; Sprague BM; Lucas SK; Torralba M; Nelson KE; Groah SL Integrated next-generation sequencing of $16 \mathrm{~S}$ rDNA and metaproteomics differentiate the healthy urine microbiome from asymptomatic bacteriuria in neuropathic bladder associated with spinal cord injury. J. Transl. Med 2012, 10, 174. [PubMed: 22929533]

(32). Keiblinger KM; Wilhartitz IC; Schneider T; Roschitzki B; Schmid E; Eberl L; Riedel K; Zechmeister-Boltenstern S Soil metaproteomics - comparative evaluation of protein extraction protocols. Soil Biol. Biochem 2012, 54, 14-24. [PubMed: 23125465]

(33). Leary DH; Hervey WJ; Li RW; Deschamps JR; Kusterbeck AW; Vora GJ Method Development for metaproteomic analyses of marine biofilms. Anal. Chem 2012, 84, 4006-4013. [PubMed: 22468925]

(34). Leary DH; Hervey WJ; Deschamps JR; Kusterbeck AW; Vora GJ Which metaproteome? The impact of protein extraction bias on metaproteomic analyses. Mol. Cell. Probes 2013, 27, 193199. [PubMed: 23831146]

(35). Tanca A; Biosa G; Pagnozzi D; Addis MF; Uzzau S Comparison of detergent-based sample preparation workflows for LTQ-Orbitrap analysis of the Escherichia coli proteome. Proteomics 2013, 13, 2597-2607. [PubMed: 23784971]

(36). Bastida F; Hernandez T; Garcia C Metaproteomics of soils from semiarid environment: functional and phylogenetic information obtained with different protein extraction methods. J. Proteomics 2014, 101, 31-42. [PubMed: 24530626]

(37). Tanca A; Palomba A; Pisanu S; Deligios M; Fraumene C; Manghina V; Pagnozzi D; Addis MF; Uzzau SA Straightforward and efficient analytical pipeline for metaproteome characterization. Microbiome 2014, 2, 49. [PubMed: 25516796]

(38). Qian C; Hettich RL Optimized extraction method to remove humic acid interferences from soil samples prior to microbial proteome measurements. J. Proteome Res 2017, 16, 2537-2546. [PubMed: 28537741]

(39). Silhavy TJ; Kahne D; Walker S The bacterial cell envelope. Cold Spring Harbor Perspect. Biol 2010, 2 (5), a000414.

(40). Edge AS; Faltynek CR; Hof L; Reichert LE; Weber P Deglycosylation of glycoproteins by trifluoromethanesulfonic acid. Anal. Biochem 1981, 118 (1), 131-137. [PubMed: 6175244]

(41). Edge ASB Deglycosylation of glycoproteins with trifluoromethanesulphonic acid: elucidation of molecular structure and function. Biochem. J 2003, 376, 339-350. [PubMed: 12974674]

(42). Chen W; Smeekens JM; Wu R Comprehensive analysis of protein N-glycosylation sites by combining chemical deglycosylation with LC-MS. J. Proteome Res 2014, 13 (3), 1466-1473. [PubMed: 24490756]

(43). Washburn MP; Wolters D; Yates JR Large-scale analysis of the yeast proteome by multidimensional protein identification technology. Nat. Biotechnol 2001, 19, 242-247. [PubMed: 11231557]

(44). Wolters DAD; Washburn MPM; Yates JRJ An automated multidimensional protein identification technology for shotgun proteomics. Anal. Chem 2001, 73 (23), 5683-5690. [PubMed: 11774908]

(45). Vizcaíno JA; Csordas A; Del-Toro N; Dianes JA; Griss J; Lavidas I; Mayer G; Perez-Riverol Y; Reisinger F; Ternent T; Xu QW; Wang R; Hermjakob H 2016 update of the PRIDE database and its related tools. Nucleic Acids Res. 2016, 44 (22), 11033-11033. [PubMed: 27683222] 
(46). Eng JK; McCormack AL; Yates JR An approach to correlate tandem mass spectral data of peptides with amino acid sequences in a protein database. J. Am. Soc. Mass Spectrom 1994, 5 (11), 976-989. [PubMed: 24226387]

(47). Xu T; Park SK; Venable JD; Wohlschlegel JA; Diedrich JK; Cociorva D; Lu B; Liao L; Hewel J; Han X; Wong CCL; Fonslow B; Delahunty C; Gao Y; Shah H; Yates JR ProLuCID: An improved SEQUEST-like algorithm with enhanced sensitivity and specificity. J. Proteomics 2015, 129, 1624. [PubMed: 26171723]

(48). Tabb DL; McDonald WH; Yates JR DTASelect and Contrast: tools for assembling and comparing protein identifications from shotgun proteomics. J. Proteome Res 2002, 1 (1), 21-26. [PubMed: 12643522]

(49). Elias JE; Gygi SP Target-decoy search strategy for increased confidence in large-scale protein identifications by mass spectrometry. Nat. Methods 2007, 4, 207-214. [PubMed: 17327847]

(50). Chatterjee S; Stupp GS; Park SKR; Ducom J-C; Yates JR; Su AI; Wolan DW A comprehensive and scalable database search system for metaproteomics. BMC Genomics 2016, 17 (1), 642. [PubMed: 27528457]

(51). Mayers MD; Moon C; Stupp GS; Su AI; Wolan DW Quantitative metaproteomics and activitybased probe enrichment reveals significant alterations in protein expression from a mouse model of inflammatory bowel disease. J. Proteome Res 2017, 16 (2), 1014-1026. [PubMed: 28052195]

(52). Moon C; Stupp GS; Su AI; Wolan DW Metaproteomics of colonic microbiota unveils discrete protein functions among colitic mice and control groups. Proteomics 2018, 18 (3-4), 1700391.

(53). Love MI; Huber W; Anders S Moderated estimation of fold change and dispersion for RNA-seq data with DESeq2. Genome Biol. 2014, 15 (12), 550. [PubMed: 25516281]

(54). Langley SR; Mayr M Comparative analysis of statistical methods used for detecting differential expression in label-free mass spectrometry proteomics. J. Proteomics 2015, 129, 83-92. [PubMed: 26193490]

(55). Ashburner M; Ball CA; Blake JA; Botstein D; Butler H; Cherry JM; Davis AP; Dolinski K; Dwight SS; Eppig JT; et al. Gene ontology: tool for the unification of biology. The Gene Ontology Consortium. Nat. Genet 2000, 25 (1), 25-29. [PubMed: 10802651]

(56). The Gene Ontology Consortium. Expansion of the Gene Ontology knowledgebase and resources. Nucleic Acids Res. 2017, 45 (D1), D331-D338. [PubMed: 27899567] 

A $\begin{gathered}\text { Sonicated and pelleted } \\ \text { microbiome sample }\end{gathered}$ (100 mg microbes) Lyophilized at $-78^{\circ} \mathrm{C}$

Incubation for $15 \mathrm{~min}$ at $4^{\circ} \mathrm{C}$

Incubation for $30 \mathrm{~min}$ at $4^{\circ} \mathrm{C}$ Neutralized

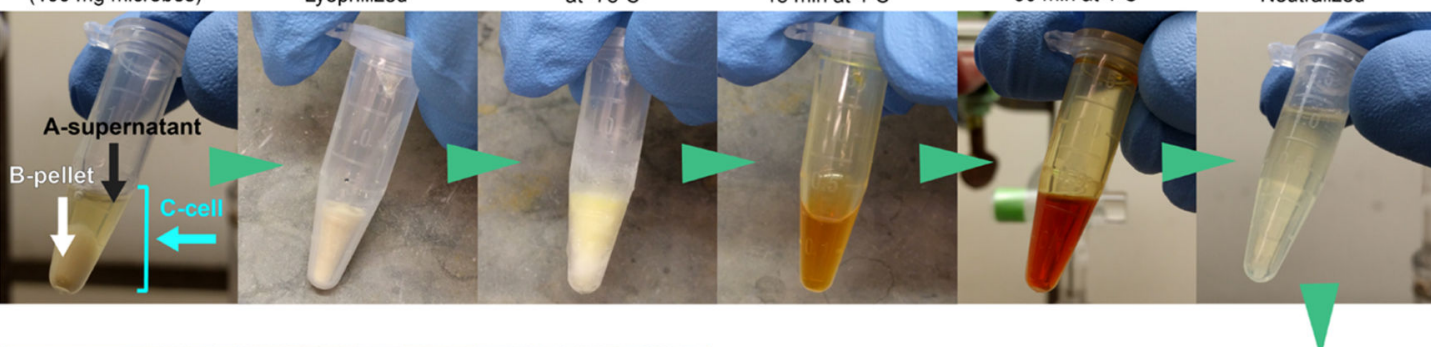

B

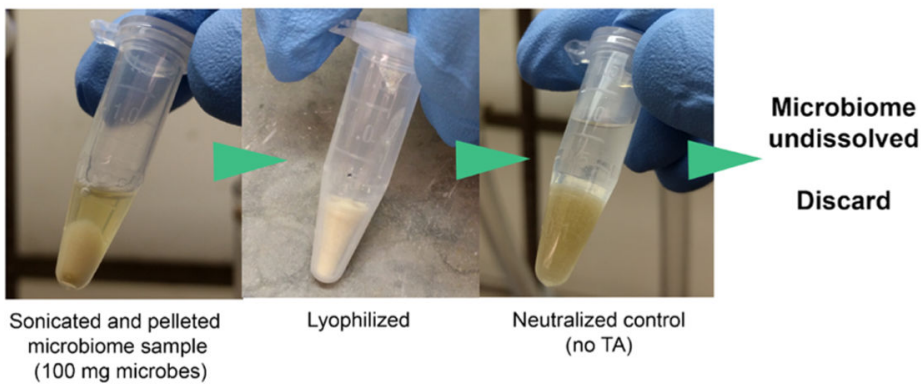

Dialyze

\section{Denature}

Reduce, Alkylate

Trypsin digest

MudPIT, LC-MS/MS

Figure 1.

Simplified proteome preparation and analysis schematic. (A) Triflic acid (TA) treatment of a microbiome sample. Note that whole bacterial cells and pellets dissolve to give a red-orange solution with TA treatment. The mixture remains soluble after neutralization (far right frame). (B) Control: treatment of lyophilized non-TA treated microbiome sample with neutralization solution. Note the remaining undissolved microbial biomass after treatment with neutralizing solution alone. 


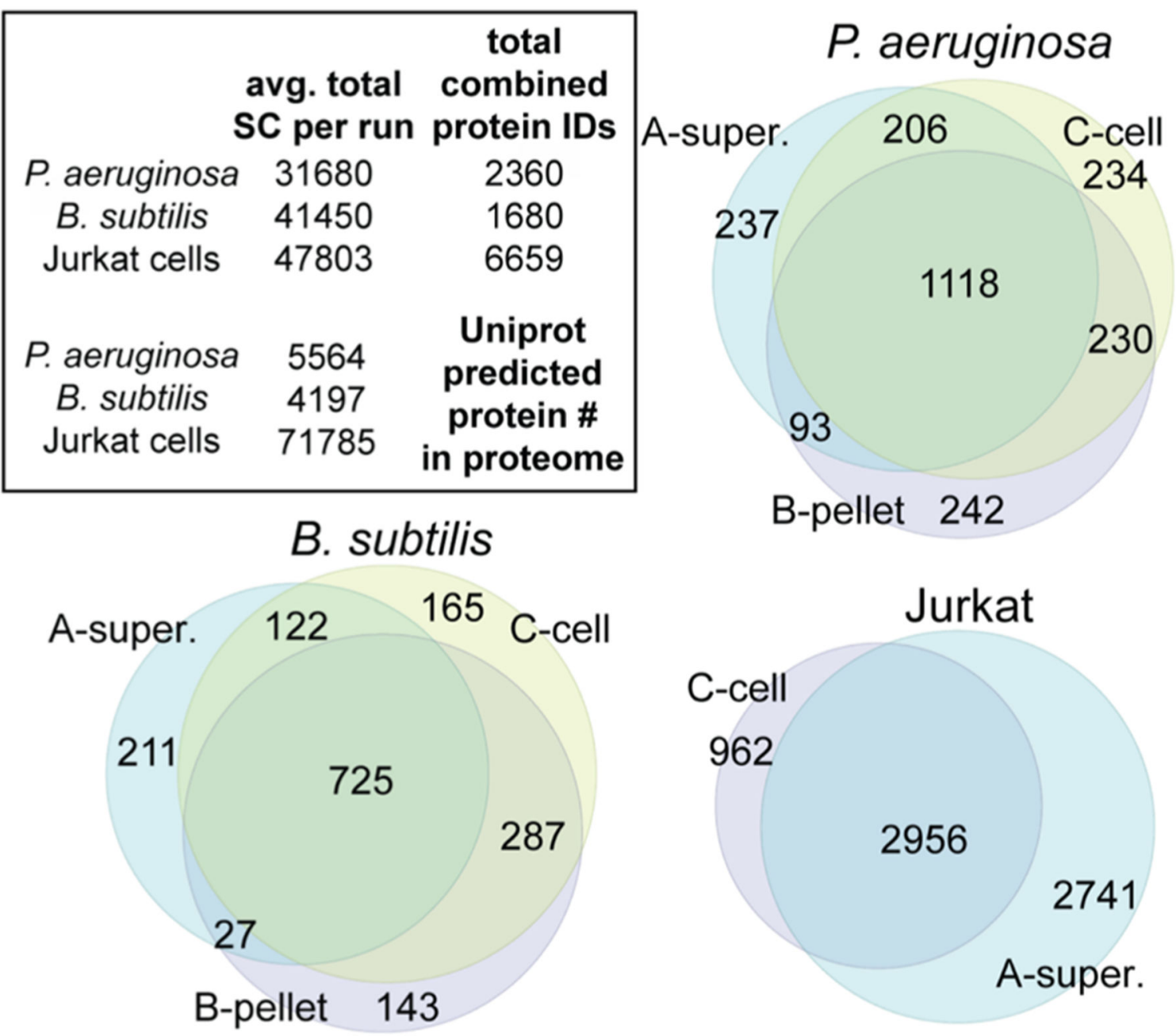

Figure 2.

Venn diagrams depict number of proteins identified by LC-MS/MS for each treatment method pertaining to $P$. aeruginosa, $B$. subtilis, and human Jurkat cells. The total average peptide spectral matches (spectral counts, SC) per LC-MS/MS run (triplicate data sets for each treatment and three cell types), total combined proteins identified per cell type, and number of predicted proteins in respective organism's genome are provided as a table in the inset. 

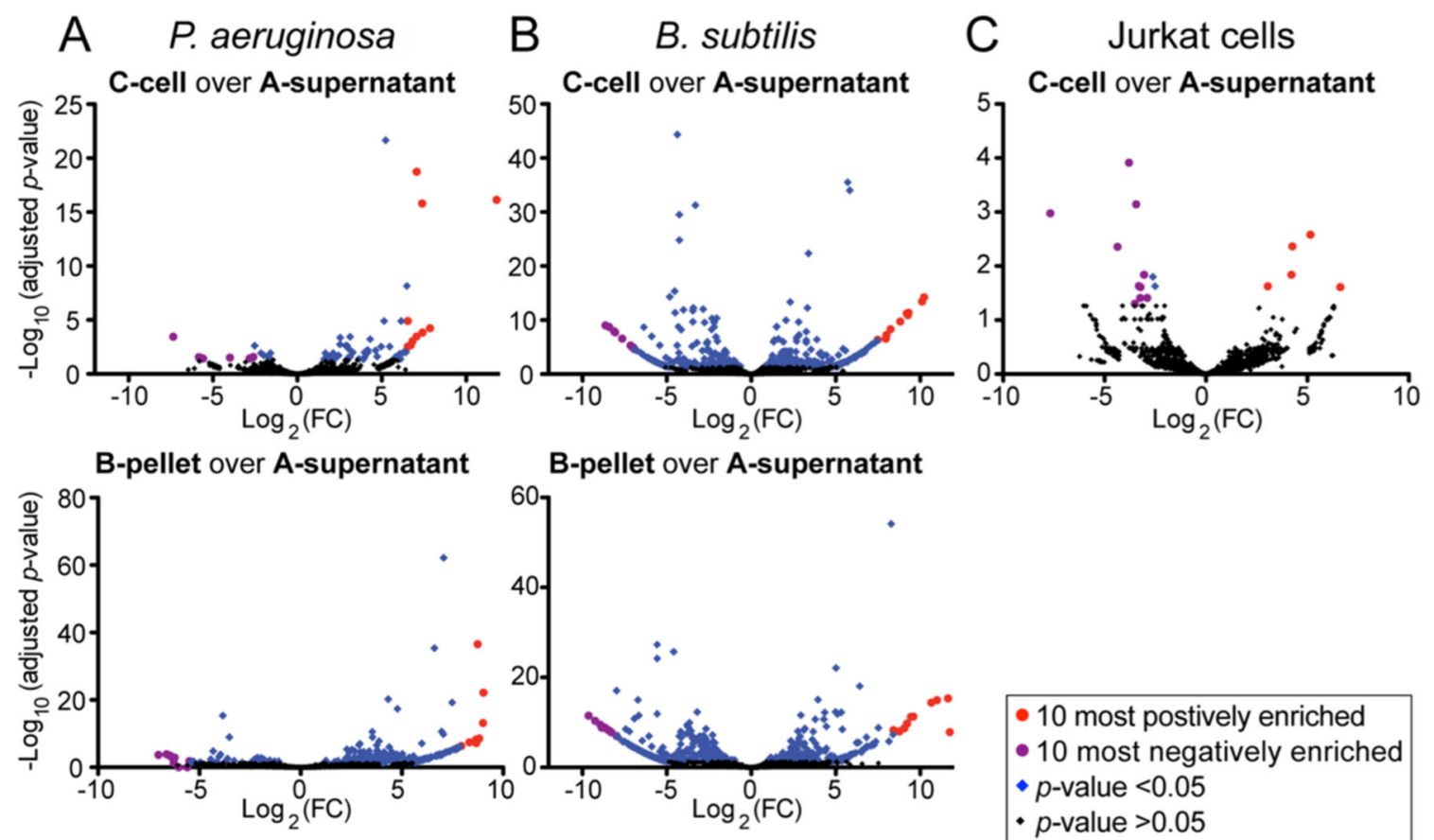

B-pellet over A-supernatant
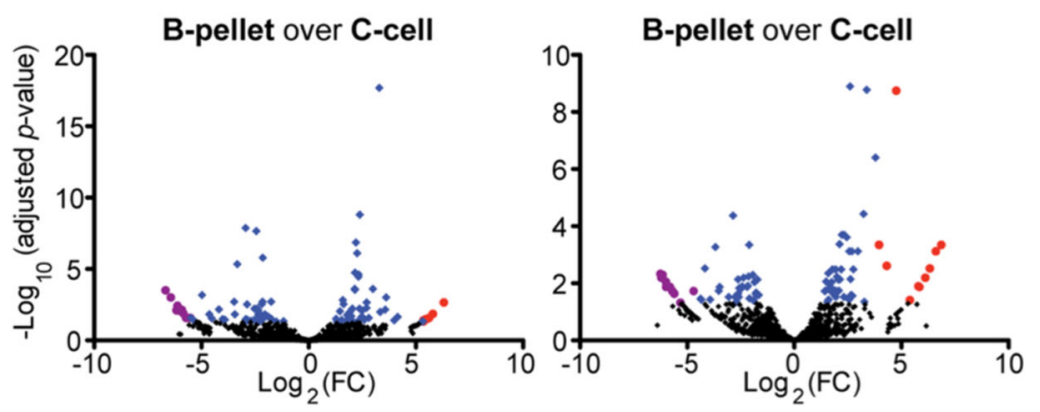

- 10 most postively enriched - 10 most negatively enriched $\bullet p$-value $<0.05$ - $p$-value $>0.05$

Figure 3.

Volcano plots depict differentially detected proteins between treatment methods for $P$. aeruginosa (A), B. subtilis (B), and human Jurkat cells (C). Significantly enriched proteins ( $p$-value $<0.05)$ are colored blue. The top 10 positively and negatively detected proteins in the B-pellet are colored red and purple, respectively. A list of the 10 most significantly differentially detected proteins is available in the Supporting Information Tables 1-10, and the complete list of proteins is in the Supporting Information data set. 
A
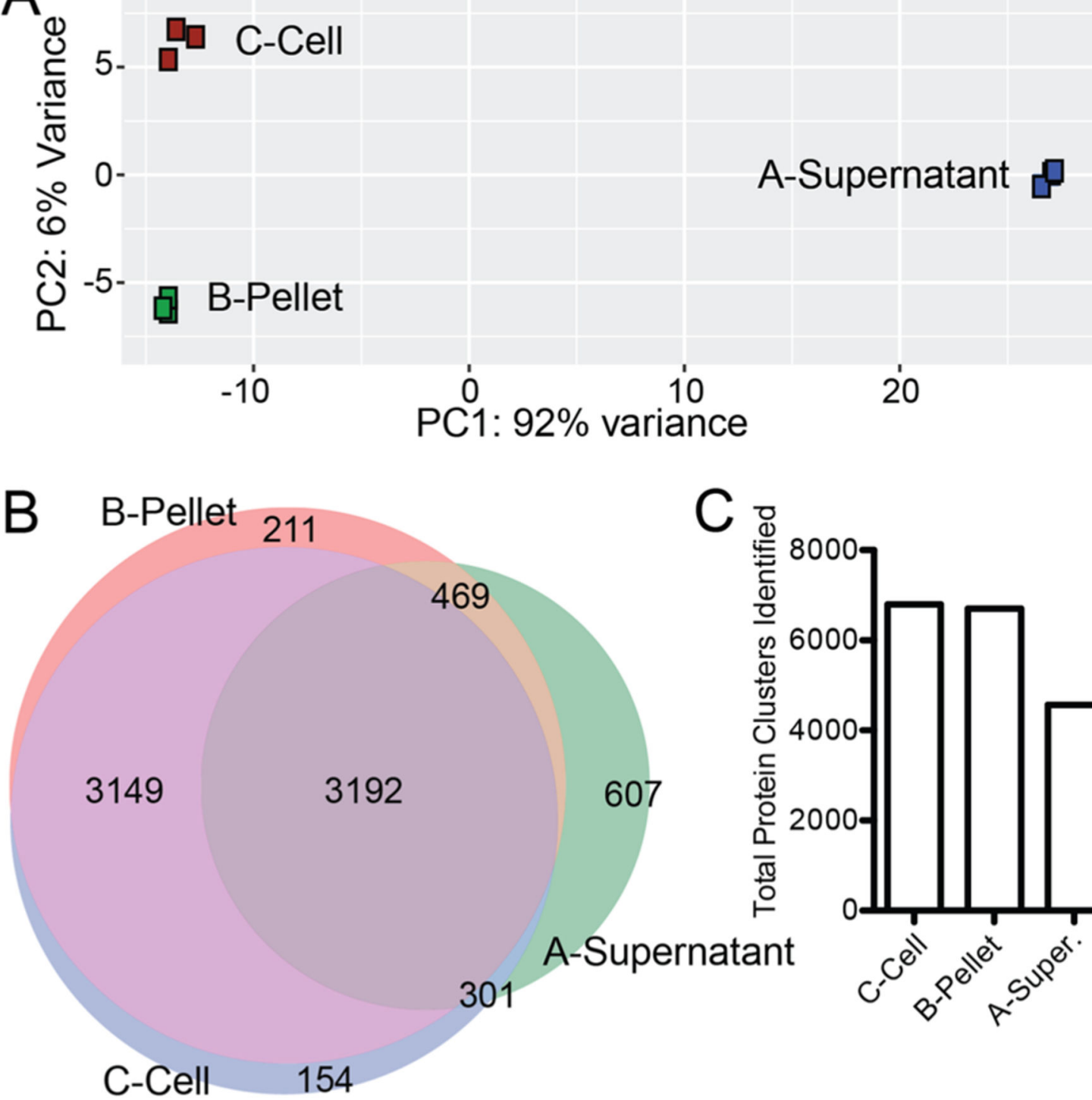

C

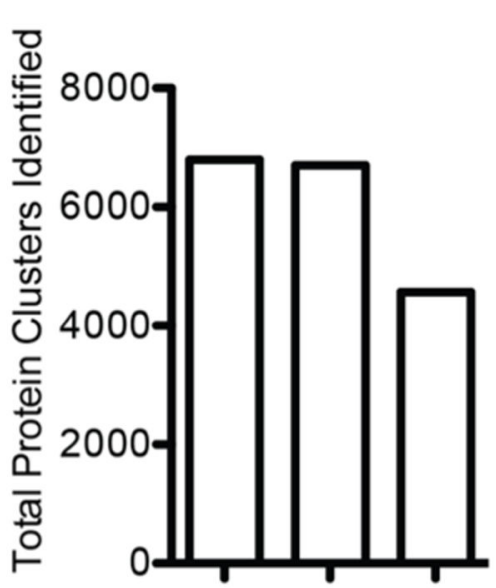

Figure 4.

Analysis of single human microbiome sample by three treatment methods. (A) Principle component analysis of LC-MS/MS-detected microbiome proteome by three treatment methods. (B) Venn diagram depicting numbers of protein clusters identified by LC-MS/MS for each treatment method. (C) Total number of protein clusters identified per treatment group. 

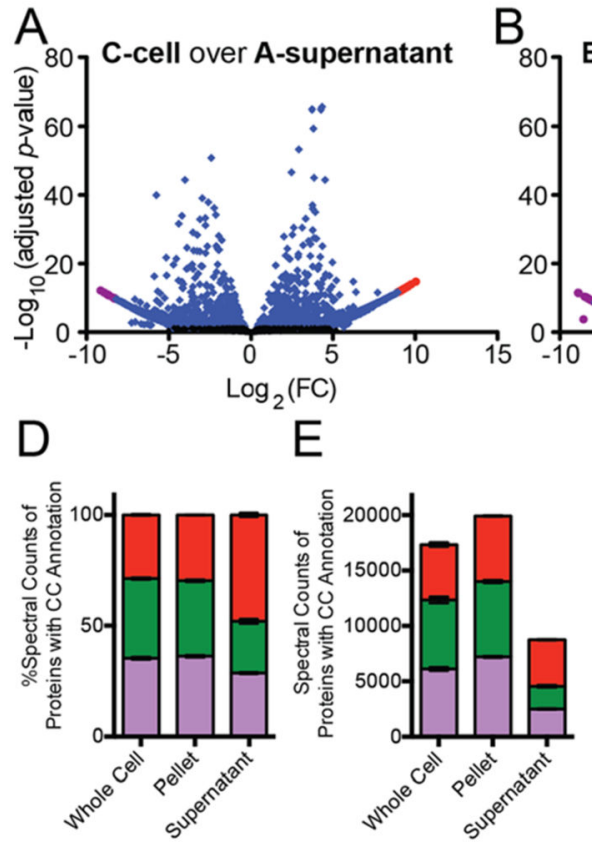

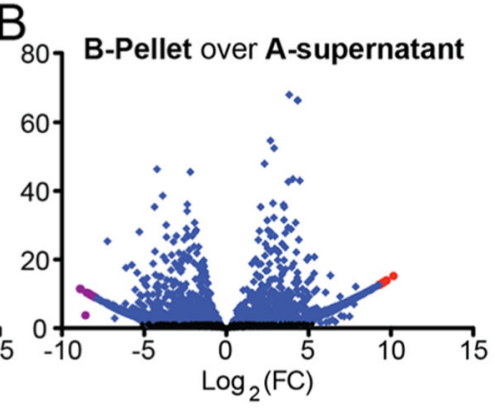

$\mathrm{F}$

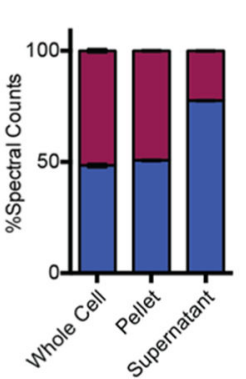

G
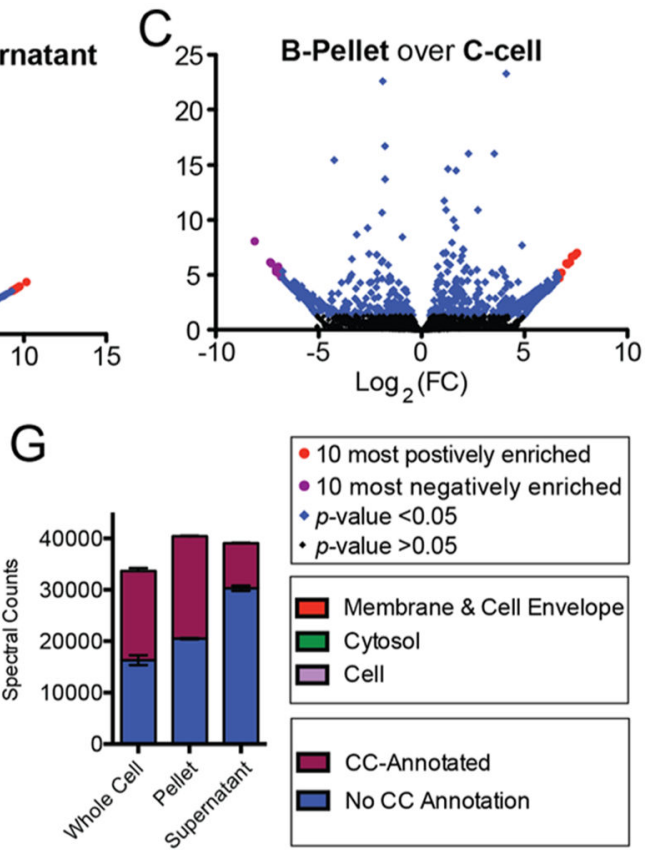

- 10 most postively enriched - 10 most negatively enriched - $p$-value $<0.05$ + $p$-value $>0.05$

$\square$ Membrane \& Cell Envelope $\square$ Cytosol $\square$ Cell

CC-Annotated

No CC Annotation

Figure 5.

Volcano plots of altered protein clusters in C-cell compared to A-supernatant (A), B-pellet compared to A-supernatant (B), and B-pellet compared to C-cell (C) for human microbiome samples. Protein clusters significantly enriched ( $p$-value $<0.05)$ are colored blue, and the top 10 positively and negatively enriched protein clusters for each volcano plot are colored red and purple, respectively. A list of the 10 most significantly differentially detected proteins is available in the Tables S11-S16 and the complete list of proteins is in the Supporting Information data set. (D,E) Cellular component GO term classification of LC-MS/MS detected microbiome proteins by treatment method. $(\mathrm{F}, \mathrm{G})$ Comparison of detected microbiome proteins with and without cellular component GO term annotations. 